\title{
OXIDATIVE DNA DAMAGE AND LIPID PEROXIDATION TO CLUSTER VS. TRADITIONAL SETS RESISTANCE EXERCISE IN PROFESSIONAL VOLLEYBALL PLAYERS
}

\author{
Hamid Arazi ${ }^{1}$, Mohsen Sahebi², and Abbas Asadi ${ }^{1,3}$ \\ ${ }^{I}$ Department of Exercise Physiology, Faculty of Sport Sciences, University of Guilan, Rasht, Iran \\ ${ }^{2}$ Department of Sport Sciences, Science and Research Branch, \\ Islamic Azad University, Guilan, Iran \\ ${ }^{3}$ Roudbar Branch, Islamic Azad University, Roudbar, Iran
}

Original scientific paper

UDC: $615.35 .03: 796.325 .015 .52$

\begin{abstract}
:
Cells continuously produce free radicals and reactive oxygen species (ROS) as part of metabolic processes. Exercise can induce an imbalance between ROS and antioxidants, which is referred to as oxidative stress. Acute bout of resistance exercise (RE) induces activation of several distinct systems of radicals generation, but the effects of different RE loading on oxidative stress response is not clear. Therefore, the purpose of this study was to examine the influence of cluster vs. traditional sets of RE on oxidative DNA damage, lipid peroxidation and uric acid response in athletes. To elicit blood oxidative stress, 12 professional young male volleyball players undertook two different RE loading patterns: 1) cluster loading pattern, 2) traditional loading pattern which was standardized for total volume and completed in a randomized crossover fashion with a four-day interval between trials. Blood samples were collected before and after RE for markers of oxidative stress and damage. In response to both the cluster and traditional sets, 8-hydroxy-2-deoxyguanosine (8-OHdG), 4-hydroxy-2-nonenal (4-HNE), and uric acid were significantly elevated post exercise $(\mathrm{p}<.05)$. Although no statistically significant differences between loading patterns were observed, the rate of elevations in 8-OHdG (effect size [ES]: 1.4 vs. 1.3) and 4-HNE (ES: 8.1 vs. 7.9) was greater for the traditional sets; likewise, the rate of uric acid excretion was greater for the cluster RE (ES: 1.6 vs. 1.4). These data suggests that RE induced DNA damage, and lipid peroxidation, but they were greater for the traditional RE. Therefore, strength and conditioning professionals in the field of volleyball must keep in their mind that RE induces oxidative stress and should use proper RE loading patterns in their training schedule.
\end{abstract}

Key words: free radicals, resistance exercise, oxidative damage biomarkers, set configuration

\section{Introduction}

Oxidative stress is a biological phenomenon marked by an imbalance between reactive free radicals (often oxygen-based molecules) and antioxidant defenses. The term oxidative stress indicates some combination of accelerated free radical production and/or exhaustion of antioxidant defenses. A severe or prolonged oxidative stress can lead to oxidatively modified lipids, proteins, and DNA (Bailey, et al., 2003; Whitehead, Olek, Fedeli, \& Falcioni, 2006). In relation to resistance training, although regular exercise has been recommended for reducing the risk of cancer and cardiovascular diseases and for other beneficial effects (Shephard \& Shek, 1998), an acute bout of resistance exercise (RE) can result in activation of several distinct systems of radicals generation (Bloomer \& Goldfarb, 2004).
It has been well documented that exercise enhances the formation of reactive oxygen species (ROS) (Radak \& Goto, 2000). Increases in oxygen consumption during exercise can enhance ROS formation (Bailey, et al., 2003) and the resulting oxidative stress (Whitehead, et al., 2006). In response to an acute or single bout of exercise, the body cannot adapt to the oxidative challenge due to the shortness of exercise duration and the physiological demands of intensity. Resistance exercise under this condition increases levels of ROS and results in oxidative damage to macromolecules (Bailey, et al., 2003; Bloomer, Goldfarb, Widem, Mckenzie, \& Consitt, 2005; Radak, Pucsok, Meeseki, Csont, \& Ferdinandy, 1999). Since ROS cause extensive DNA damage, including single-strand breaks and the formation of modified bases (Halliwell \& Aruoma, 
1991). One of the abundant forms of oxidized DNA and lipid peroxidation are 8-hydroxy-2-deoxyguanosine (8-OHdG) and 4-hydroxy-2-nonenal (4-HNE), respectively. It has been shown that maximal intensity of exercise induces elevation of oxidative stress (Benitez-Sillero, et al., 2011; Cuevas, et al., 2005; Quindry, Stone, King, \& Broeder, 2003; Radak, et al., 2003). However, several researchers indicated that exhaustive RE (McAnulty, et al., 2005) and strenuous bouts of squat or cycle sprint exercises (Bloomer, et al., 2005) did not induce oxidative stress in trained subjects. In relation to DNA oxidation, previous authors assessed 8-OHdG response, as a marker of DNA oxidation, to isotonic resistance exercise (Bloomer, et al., 2005). An increase in 8 -OHdG of the quadriceps muscle was reported at the time point 24 hours after 200 eccentric actions of the knee extensors (Radak, et al., 1999), whereas no change was reported in 8-OHdG after 30-minute dumbbell squat with $70 \%$ of 1 RM (Bloomer, et al., 2005).

Current literature about RE and oxidative stress response is well described, whereas there is no available data about the differences between RE loading patterns in oxidative stress. In comparison to previous studies that used traditional sets (performing multiple repetitions in a set with no rest between the repetitions), a new approach for loading pattern in RE called "cluster sets" (Haff, et al., 2008; Lawton., et al., 2004; Lawton, et al., 2006) was used.

Cluster loading, sometimes termed inter-repetition rest training, describes a training system whereby rest periods are manipulated so as to break sets into small clusters of repetitions (Haff, et al., 2008; Lawton, et al., 2004; Lawton et al., 2006). Cluster sets are theorized to allow for some replenishment of phosphocreatine (Lawton, et al., 2006), which is vital for power production (Kraemer \& Ratamess, 2004; Weiss, 1991; Williams, Natalie, Louise, \& Jeff, 2006). Also, Baker and Newton (2005) asserted that it is important to avoid fatigue when attempting to maximize power, as well as that performing a low amount of repetitions, with an appropriate rest intervals, will optimize power; however, the oxidative stress responses to this type of loading pattern, which is optimal for improving power and strength performance (Haff, et al., 2008), is not clear.

Overall, DNA damage and lipid peroxidation following a session of RE have been reported in previous studies, but the influence of RE with different loading patterns (traditional vs. cluster) on human subjects are not clear. Therefore, the aim of this study was to investigate the effects of an acute RE with different loading patterns on blood markers of oxidative damage. This approach was used to demonstrate: (a) changes in oxidative stress (8-OHdG and 4-HNE), and (b) changes in uric acid.
We hypothesized that (a) cluster and traditional sets will produce increases in 8-OHdG, 4-HNE and uric acid, and (b) the traditional sets will produce greater increases in oxidative stress than cluster sets.

\section{Methods}

\section{Study design}

To compare the effects of cluster vs. traditional sets of RE on oxidative modification of DNA and lipids, 12 young male national team volleyball players volunteered to participate in this study and performed RE with randomized crossover fashion with four days interval between trials. The blood samples were drawn before (baseline-test) and after the RE (posttest) for the measurement of (a) 8-OHdG, (b) 4-HNE, and (c) uric acid. The participants undertook the RE between 11:00 and 13:00 hours (in a weight training facility). The participants were also instructed to avoid any strenuous physical activity and to maintain their usual dietary habits throughout the duration of the study.

\section{Participants}

Twelve professional young male volleyball players, who were members of the national team, with experience in resistance training and plyometric training, volunteered to participate in this study. The participants were free from any lower and upper body injuries, cardiovascular and orthopedic diseases. The method and procedure used in this study was approved by the Institutional Review Board for use of human subjects in research. The experiment was conducted according to the Declaration of Helsinki. Before data collection, the participants were informed about the benefit and possible risks associated with the study. Afterwards they provided written informed consent. Also, informed written consent was obtained from the parents or legal guardians when the participants were under aged. Participants' characteristics are presented in Table 1.

Table 1. Participants' characteristics

\begin{tabular}{lcccc}
\hline & $\mathrm{N}$ & $\mathrm{M} \pm \mathrm{SD}$ & $\mathrm{Min}$ & $\mathrm{Max}$ \\
\hline Age (year) & 12 & $17.83 \pm 1.03$ & 16 & 19 \\
Body mass $(\mathrm{kg})$ & 12 & $79.75 \pm 5.53$ & 70 & 86 \\
Body height $(\mathrm{cm})$ & 12 & $194 \pm 6.35$ & 182 & 200 \\
BMl $\left(\mathrm{kg} / \mathrm{m}^{2}\right)$ & 12 & $21.03 \pm 1.85$ & 17.5 & 24.1 \\
\hline
\end{tabular}

\section{Procedures}

Athletes involved in this study performed two RE loading patterns (cluster vs. traditional). Totally, the participants reported to the laboratory four times. On day one, subjects' body height was measured using a wall-mounted stadiometer (Seca 


\begin{tabular}{|c|c|c|c|c|c|}
\hline Day 1 & Day 2 & Interval & Day 3 & Interval & Day 4 \\
\hline $\begin{array}{c}\text { Body height and mass } \\
\text { measurements+Familiarization } \\
\text { with exercise }\end{array}$ & 1RM testing & 10 days & $\begin{array}{l}\text { Blood sampling+Cluster loading+Blood } \\
\text { sampling }\end{array}$ & 4 days & $\begin{array}{l}\text { Blood sampling+Traditional loading+Blood } \\
\text { sampling }\end{array}$ \\
\hline
\end{tabular}

Figure 1. Study design.

0123, Germany) to the nearest centimeter. Body mass was measured to the nearest $0.1 \mathrm{~kg}$ using a medical scale (Seca 760102, Germany). Also, during this session the participants were familiarized with REs selected for the study. On day two, the one repetition maximum (1RM) of each resistance exercise was measured. Ten days after the 1RM testing, the athletes reported to the laboratory and performed cluster sets (day 3) and traditional sets (day 4) RE with 96 hours interval between the experimental sessions. Before and after the exercise sessions blood samples were collected to analyze oxidative stress responses (Figure 1). All sessions were held from 11:00 a.m. until 1:00 p.m. The athletes were instructed to observe the following recommendations before the exercise sessions: (a) take a light meal two hours before the experiments, (b) not to do any type of physical activity within 48 hours before the test, (c) abstain from alcohol, caffeinated drinks or stimulants for 24 hours, and (d) maintain habitual dietary intake at least seven days prior to testing and during the study period. The ambient temperature was fixed at $27 \pm 1 \mathrm{C}^{\circ}$ and the air humidity during the tests ranged between $60 \%$ and $70 \%$. An experienced laboratory technician phlebotomist performed the blood sampling before and after RE for all the subjects.

\section{RM testing}

The 1RM testing was performed according to method previously described by Kraemer and Fry (1995). After a general warm-up including five-minute cycling on a cycle-ergometer (Sport Art Fitness, C52u, Taiwan) and five minutes of various stretching exercises, the participants performed a warm-up set of five repetitions with $30 \%$ of $1 \mathrm{RM}$. The second warm-up consisted of a set of four repetitions with $50 \%$ of $1 \mathrm{RM}$, and the third warm-up included four repetitions with $70 \%$ of $1 \mathrm{RM}$. The fourth trial consisted of one repetition with $90 \%$ of $1 \mathrm{RM}$. After the warm-up, each athlete was tested for the 1RM by increasing the load during consecutive trials until the participants were unable to perform a proper lift or a complete range of motion with correct technique. The 1RM test was determined by three sets of one repetition, with 2- to 4-minute rests among attempts. Spotters were present to provide verbal encouragement and safety for the subjects.

\section{Cluster and traditional sets}

Before each exercise session loading pattern, the participants performed a 10-minute warm-up including 5-minute cycling on a cycle-ergometer (Sport Art Fitness, C52u, Taiwan) at a self-selected workload and cadence, as well as around five minutes of various stretching exercises with the aim of preparing joints for the REs. Then participants performed cluster and traditional loading pattern in a randomized-order fashion. The RE order was back squat, clean pull, power clean and jump squat, respectively. The cluster loading pattern consisted of four trials for each exercise in this manner: first, one set of eight repetitions with $80 \%$ of $1 \mathrm{RM}$ for the back squat, clean pull and power clean with 180 second rest intervals between the sets. Trial two consisted of $2 \times 4$ repetitions with $80 \%$ of $1 \mathrm{RM}$ with rest intervals of 30 and 120 seconds. Trial three consisted of $2 \times 3$ repetitions with $85 \%$ of $1 \mathrm{RM}$ with rest intervals of 30 and 120 seconds. At the end, the subjects performed $2 \times 2$ repetitions with $90 \%$ of $1 \mathrm{RM}$ with rest intervals of 30 and 120 seconds. In jump squat exercise, the participants performed 5 repetitions with $30 \%$ of $1 \mathrm{RM}$ in trial one and then performed $2 \times 2$ repetitions with decreasing intensity. In contrast, the traditional loading pattern consisted of four trials for each exercise with decreases in repetitions and increases in exercise intensity (Hansen, Cronin, Pickering, \& Newton, 2011). The details of each exercise program are presented in Table 2.

\section{Blood sampling}

Blood samples were collected before and after each RE session (cluster and traditional). Blood samples were drawn $(3 \mathrm{ml})$ from the antecubital vein into plain evacuated test tubes. Then the blood was collected into EDTA-containing tubes and centrifuged immediately at $1370 \times \mathrm{g}$ for 10 min at $4^{\circ} \mathrm{C}$, and the plasma was collected. The blood was allowed to clot at room temperature for 30 minutes and centrifuged at $1500 \times \mathrm{g}$ for 10 minutes. The serum layer was removed and frozen at $-20^{\circ} \mathrm{C}$ in multiple aliquots for further analyses. Serum 8-OHdG, 4-HNE and UA were measured following the manufacturer's protocol. The respective intra- and interassay CVs were $3.5 \%$ and $4.2 \%$ for $8-\mathrm{OHdG}, 3.7 \%$ and $4.9 \%$ for $4-\mathrm{HNE}$, and $4.3 \%$ and $4.8 \%$ for UA. The serum $8-\mathrm{OHdG}$ level was measured by an ELISA using an 8-OHdG Assay Kit (EASTBIOPHARM Co., Ltd, China) according to the manufacturer's instruction. 8-OHdG is an important index of DNA oxidative damage following oxidative stress.

The serum 4-HNE levels were measured with available kit (EASTBIOPHARM Co., Ltd, China) 
Table 2. Cluster and traditional resistance exercise program

\begin{tabular}{|c|c|c|c|c|c|c|c|c|c|c|c|c|}
\hline & \multicolumn{12}{|c|}{ Cluster } \\
\hline & \multicolumn{3}{|c|}{ Trial 1} & \multicolumn{3}{|c|}{ Trial 2} & \multicolumn{3}{|c|}{ Trial 3} & \multicolumn{3}{|c|}{ Trial 4} \\
\hline & Reps & $\begin{array}{l}\% \text { of } \\
1 \mathrm{RM}\end{array}$ & Rest (s) & Reps & $\begin{array}{l}\% \text { of } \\
1 \mathrm{RM}\end{array}$ & Rest (s) & Reps & $\begin{array}{l}\% \text { of } \\
1 \mathrm{RM}\end{array}$ & Rest (s) & Reps & $\begin{array}{l}\% \text { of } \\
1 \mathrm{RM}\end{array}$ & Rest (s) \\
\hline $\begin{array}{l}\text { Back } \\
\text { squat }\end{array}$ & 8 & 80 & 180 & $2 \times 4$ & 80 & $30 / 120$ & $2 \times 3$ & 85 & $30 / 120$ & $2 \times 2$ & 90 & $30 / 120$ \\
\hline $\begin{array}{l}\text { Clean } \\
\text { pull }\end{array}$ & 8 & 80 & 180 & $2 \times 4$ & 80 & $30 / 120$ & $2 \times 3$ & 85 & $30 / 120$ & $2 \times 2$ & 90 & $30 / 120$ \\
\hline $\begin{array}{l}\text { Power } \\
\text { clean }\end{array}$ & 8 & 80 & 180 & $2 \times 4$ & 80 & $30 / 120$ & $2 \times 3$ & 85 & $30 / 120$ & $2 \times 2$ & 90 & $30 / 120$ \\
\hline \multirow[t]{4}{*}{$\begin{array}{l}\text { Jump } \\
\text { squat }\end{array}$} & 5 & 30 & $30 / 180$ & $2 \times 2$ & 20 & $30 / 120$ & $2 \times 2$ & 10 & $30 / 120$ & $2 \times 2$ & 0 & $30 / 120$ \\
\hline & \multicolumn{12}{|c|}{ Traditional } \\
\hline & \multicolumn{3}{|c|}{ Trial 1} & \multicolumn{3}{|c|}{ Trial 2} & \multicolumn{3}{|c|}{ Trial 3} & \multicolumn{3}{|c|}{ Trial 4} \\
\hline & Reps & $\begin{array}{l}\% \text { of } \\
1 \mathrm{RM}\end{array}$ & Rest (s) & Reps & $\begin{array}{l}\% \text { of } \\
1 \mathrm{RM}\end{array}$ & Rest (s) & Reps & $\begin{array}{l}\% \text { of } \\
1 \mathrm{RM}\end{array}$ & Rest (s) & Reps & $\begin{array}{l}\% \text { of } \\
1 \mathrm{RM}\end{array}$ & Rest (s) \\
\hline $\begin{array}{l}\text { Back } \\
\text { squat }\end{array}$ & 8 & 80 & 180 & 8 & 80 & 180 & 6 & 85 & 180 & 4 & 90 & 180 \\
\hline $\begin{array}{l}\text { Clean } \\
\text { pull }\end{array}$ & 8 & 80 & 180 & 8 & 80 & 180 & 6 & 85 & 180 & 4 & 90 & 180 \\
\hline $\begin{array}{l}\text { Power } \\
\text { clean }\end{array}$ & 8 & 80 & 180 & 8 & 80 & 180 & 6 & 85 & 180 & 4 & 90 & 180 \\
\hline $\begin{array}{l}\text { Jump } \\
\text { squat }\end{array}$ & 5 & 30 & 180 & 4 & 20 & 180 & 4 & 10 & 180 & 3 & 0 & 180 \\
\hline
\end{tabular}

following the manufacturer's protocol. 4-HNE is an $\alpha, \beta$-unsaturated hydroxyalkenal produced by lipid peroxidation in cells. 4-HNE is the primary $\alpha, \beta$-unsaturated hydroxyalkenal formed in this process. It can be found throughout animal and human tissues and in higher quantities during oxidative stress due to the increase in the lipid peroxidation chain reaction, due to the increase in stress events (Bloomer, et al., 2005).

The serum uric acid (UA) was measured spectrophotometrically using UA kites provided from SPINREACT. Elevation of UA after exercise usually parallels total antioxidant capacity changes and has been estimated to account for nearly one-third of total antioxidant capacity increase.

\section{Statistical analyses}

Descriptive statistics $(\mathrm{M} \pm \mathrm{SD})$ were reported for all the dependent variables. Data normality was verified by the Kolmogorov-Smirnoff test; therefore, a nonparametric test was not necessary. A $2 \times 2$ (cluster vs. traditional and pre vs. post) analysis of variance was used to analyze data. Bonfferoni's post-hoc analyses were used to determine pairwise differences when significant main effects or interactions were observed. The magnitude of changes from pre- to post-exercise was assessed by effect size (ES; the difference between the pre-test and post-test scores divided by the pre-test standard deviation). The level of significance was set at $p \leq .05$. The statistical tests were performed using the SPSS statistical package, version 16 (Chicago, IL, USA).

\section{Results}

The results show that both the cluster and traditional loading patterns significantly increased 8 -OHdG (from $1.04 \pm 0.78$ to $2.06 \pm 0.4$ and from $1.01 \pm 0.74$ to $2.05 \pm 0.51$, respectively; $\mathrm{F}=23.32$, $\mathrm{p}=.001$ ); 4-HNE (from $1.12 \pm 0.62$ to $1.61 \pm 0.58$ and from $1.01 \pm 0.59$ to $1.49 \pm 0.57: \mathrm{F}=5.66, \mathrm{p}=.026$, respectively) and UA (from $4.8 \pm 0.78$ to $6.05 \pm 0.88$ and from $4.41 \pm 0.92$ to $5.74 \pm 0.85: \mathrm{F}=112.47, \mathrm{p}=.001$, respectively) in comparison to the pre-exercise values. There were no differences between the cluster and traditional loading patterns in the 8-OHdG $\left(\mathrm{F}_{1,22 \mathrm{GG}}=0.004, \mathrm{p}=.951\right), 4-\mathrm{HNE}$ $\left(\mathrm{F}_{1,22 \mathrm{GG}}=0.001, \mathrm{p}=.976\right)$ and UA $\left(\mathrm{F}_{1,22 \mathrm{GG}}=0.119\right.$, $\mathrm{p}=.734)$; however, the rate of ES was greater for the traditional sets in the $8-\mathrm{OHdG}$ (1.4 vs. 1.3) and 4-HNE ( 0.81 vs. 0.79$)$; likewise, the rate of ES was greater for the cluster sets in the UA (1.6 vs. 1.4) (Figure 2).

\section{Discussion and conclusions}

The aim of this study was to investigate the effects of different RE loading patterns on the oxidative stress response. The results indicated that both the cluster and traditional exercise sets increased blood oxidative stress; however, the increases were greater for the traditional loading pattern. The findings are in line with our hypothesis 


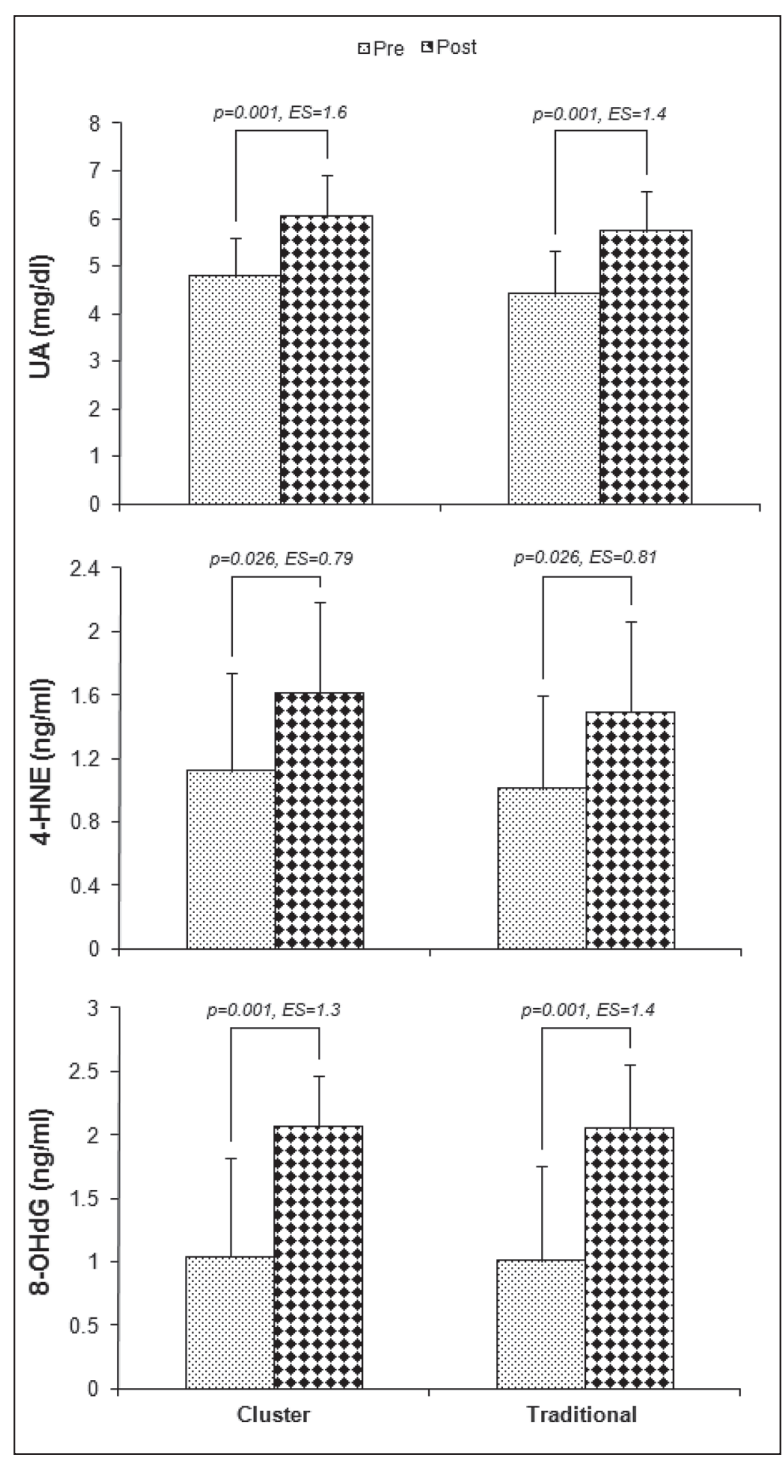

Figure 2. Pre-to-post resistance exercise changes in 8-OHdG, 4-HNE, and uric acid (UA). Values are $M \pm S D$.

that oxidative stress caused by the traditional sets would be higher despite the fact that these differences were not significant.

Energy consumption and oxygen need increases during RE. Free radicals are formed as by-products of the normal metabolism, while it has been suggested that the production of ROS would increase as the result of the increased oxygen consumption by working muscles (Bailey, et al., 2003; Whitehead, et al., 2006). Cellular DNA damage can be caused by ROS generated under different conditions such as RE. In this study, the 8-OHdG concentration significantly increased post RE for both the cluster and traditional loading patterns with the latter being slightly greater (1.4 vs. 1.3), which was in agreement with previous studies that reported that DNA damage enhanced following marathon race, aerobic and/or anaerobic exercise, and acute strenuous exercise (Bloomer, et al., 2006; 2004; Demirba, et al., 2006; Pozzi, et al., 2010; Sakai, et al., 1999; Quindry, et al., 2003; Wierzba, Olek,
Fedeli, \& Falcioni, 2006). Paradoxically, in a study conducted by Bloomer and Fisher-Wellman (2008), the relation between blood oxidative stress markers and gender, training status and dietary content were examined and no significant differences in the 8-OHdG levels were determined. In contrast to our findings, Bloomer et al. (2005) did not find significant changes in the 8 -OHdG concentration after the 30-minute dumbbell squat exercise. Possible explanations for the discrepancy between our findings and those of the previous studies that reported no exercise effects on oxidative stress could be type of exercise and RE intensity (Bloomer, et al., 2005; Bloomer \& Goldfarb, 2004).

Although the exact mechanism of producing ROS via RE is not clear, Uchiyama, Tsukamoto, Yoshimura, and Tamaki (2006) reported that a relatively anoxic state occurred repeatedly after every RE set in the exercising muscle following rapid blood reperfusion and it was similar to an ischemia-reperfusion state. It seems this ischemiareperfusion state induced ROS production and, consequently, 8-OHdG activity and DNA damage. The other possible mechanisms responsible for the RE-induced ROS formation include xanthine-xanthine oxidase pathway, respiratory burst of neutrophils, catecholamine autooxidation, local muscle ischemia-hypoxia, conversion of the weak superoxide to the strong hydroxyl radical by lactic acid, and alteration of calcium homeostasis (Ji, 2000).

In our study, we did not find significant differences between the cluster and traditional loading patterns in 8-OHdG activity, but the rate of production was slightly greater for the traditional (1.4 vs. 1.3). It seems that configuration of the traditional sets, as compared to the cluster sets, and assigned rest intervals between repetitions can induce oxygen availability for the active muscles, preventing to produce greater metabolic status (e.g., $\mathrm{H}^{+}$) and lactate concentrations (Haff, et al., 2008) and, consequently, curb the $8-\mathrm{OHdG}$ production; however, this note could be speculation and further studies are necessary in this area.

In addition to oxidative DNA damage, free radicals may change cellular components, especially lipids, and lead to the initiation of chain reactions that are known as lipid peroxidation, being the most important consequence of oxidative stress (Uchida \& Stadtman, 1992; Houstis, Rosen, \& Lander, 2006). 4 -HNE is a lipid peroxidation product that is released from the peroxidation of $n-6$ polyunsaturated fatty acids; it covalently modifies proteins on their cysteine, histidine and lysine residues, thereby modulating the activity of several proteins (Uchida \& Stadtman, 1992). 4-HNE, an end product of lipid peroxidation, is widely recognized as a specific marker of oxidative stress being increased by increments in ROS leveles (Houstis, et al., 2006). In this study, 4-HNE concentration significantly increased 
post RE for both the cluster and traditional loading patterns, with a slightly greater change for the traditional one ( 0.81 vs. 0.79). The increases in lipid peroxidation following RE is in accordance with the results of Ramel, Wagner, and Elmadfa (2004) who addressed statistically significant enhancements in lipid peroxidation after circuit-type RE in trained and untrained subjects. An increase in 4-HNE excretion is in line with increases in intracellular ROS production in L6 muscle cells (Pillon, et al., 2012). The resulting redox imbalance creates ROS that induces lipid peroxidation, thus generating aldehydes that generate more ROS, leading to amplification of the damage caused by oxidative stress (Bloomer, et al., 2006). The other possible mechanisms of increases in the 4-HNE concentration after RE may be muscle damage and increase in creatine kinase (CK) activity (Nikolaidis \& Mougios, 2004). Previous authors reported positive relationship between CK activity and lipid peroxidation after RE. This relationship between muscleenzyme release and oxidative damage might have resulted from an increase in membrane permeability due to lipid peroxidation and/or increased membrane permeability rendering polyunsaturated fatty acids of membrane more susceptible to peroxidation (Nikolaidis \& Mougios, 2004).

Since cellular membranes are rich in polyunsaturated fatty acids, increased damage of muscle cells after RE can potentially lead to the increased concentration of polyunsaturated fatty acids in the blood (Nikolaidis \& Mougios, 2004). Considering that unsaturated fatty acids are much susceptible to lipid peroxidation, this is probably one of the mechanisms through which RE increases lipid peroxidation after exercise in the blood (Foley, Jayaraman, \& Prior, 1999; Hulbert, 2005); however, we did not directly measure the muscle damage indicators (previous studies reported these), thus it could be a speculation.

Uric acid elevation post-exercise usually parallels total antioxidant capacity changes (Margonis, et al., 2007); UA elevation has been estimated to account for nearly one-third of total antioxidant capacity increase (Whitehead, et al., 1992). In our study, UA excretion significantly increased post RE in both the cluster and traditional loading patterns with a slightly greater increment for the cluster one (1.6 vs. 1.4), which is in agreement with previous studies reporting enhanced UA post RE (Clarkson, Kearns, Rouzier, Rubin, \& Thompson, 2006). High-intensity exercise-associated muscle ischemia regulates purine nucleotide metabolism leading to adenosine elimination of monoposphate adenosine (AMP) and accumulation of hypoxanthine in the muscle component and plasma. Although hypoxanthine may be converted back to AMP at rest and during mild exercise, it is converted to UA and oxygen radicals (MaClaren \& Morton, 2012).
Elevation of UA in our study is in line with those observations. The involvement of purine nucleotide metabolism during RE indicates an ATP reduction and IMP rise in muscle after RE. The minimal increases (not significant) in UA elevation for the cluster loading pattern, as compared with the traditional loading pattern, could be a higher involvement of purine nucleotide metabolism caused by the assigned inter-set rest intervals and, consequently, performing RE with more muscle involvements.

Overall, in our study we found that responses of oxidative stress were lower for the cluster loading pattern, whereas UA changes were higher for the cluster sets in comparison to the traditional loading pattern. Possible explanations for these responses could be brief rest interval between sets of cluster RE. Rest intervals between sets provide athletes with opportunities for metabolic recovery, allow them to manage fatigue, facilitate partial restoration of cellular environment, and replacement of PCr. It seems that lower oxidative stress responses and greater UA changes, caused by rest intervals between sets in the cluster loading pattern sets, make this pattern slightly better than the traditional sets to curb the production of oxidative damage and enhancement of antioxidant status.

In conclusion, this study suggests that RE would provide a stimulus to enhance oxidative stress in volleyball players. The results also suggest that the oxidative DNA damage and lipid peroxidation may be sensitive to a type of RE loading pattern and rest intervals between exercise sets because the volleyball players performing RE cluster loading pattern experienced lower increases than when performing the traditional loading pattern RE. Strength and conditioning coaches in the field of volleyball must keep in their mind that RE can induce oxidative stress that should be decreased or regulated. Therefore, it can be recommended that strength and conditioning professionals should use cluster loading pattern RE for volleyball players to reduce oxidative stress following a session of resistance training. Further studies are necessary on the use of antioxidant supplements and in order to determine oxidative stress responses to different types of loading patterns of RE. The exact mechanisms by which the cluster loading pattern induces lower enhancement of oxidative stress are not elucidated. However, the important causes of ROS include $\mathrm{H}_{2} \mathrm{O}_{2}, \mathrm{O}_{2}$, and possibly $\mathrm{OH}_{2}$ and peroxynitrite in skeletal muscles, which commonly induce oxidative DNA damage and lipid peroxidation after RE.

However, the results of the present study must be interpreted with caution since the subjects were a selected group of young male volleyball players. Generalization of the results to other players would, therefore, not be accurate. Another limitation that needs to be considered is a small number of subjects. Small group size in this study could have 
caused outliers to influence the mean values of the respective oxidative stress values more than would have been the case with a larger group size. In light of the afore-mentioned factors, further studies in the area of RE with differing loading patterns are needed to test the changes of oxidative stress among a much bigger sample size of athletes from different sports disciplines. These studies should also conduct biochemical and biomechanical analysis to identify the precise neural and musculoskeletal mechanisms that underlie the changes in the oxidative stress after a RE session.

\section{References}

Bailey, D.M., Davies, B., Young, I.S., Jackson, M.J., Davison, G.W., Isaacson, R., \& Richardson, R.S. (2003). EPR spectroscopic detection of free radical outflow from an isolated muscle bed in exercising humans. Journal of Applied Physiology, 94, 1714-1718.

Baker, D., \& Newton, R.U. (2005). Methods to increase the effectiveness of maximal power training for the upper body. Journal of Strength and Conditioning Research, 27, 24-32.

Benitez-Sillero, J.D., Perez-Navero, J.L., Tasset, I.I., Guillen-Del Castillo, M., Gil-Campos, M., \& Tunez, I. (2011). Cardiorespiratory fitness and oxidative stress: Effect of acute maximal aerobic exercise in children and adolescents. Journal of Sports Medicine and Physical Fitness, 51, 204-210.

Bloomer, R.J., Falvo, M.J., Fry, A.C., Schilling, B.K., Smith, W.A., \& Moore, C.A. (2006). Oxidative stress response in trained men following repeated squats or sprints. Medicine and Science in Sports and Exercise, 38, 1436-1442.

Bloomer, R.J., \& Fisher-Wellman, K.H. (2008). Blood oxidative stress biomarkers: Influence of sex, exercise training status, and dietary intake. General Medicine, 5, 218-228.

Bloomer, R.J., \& Goldfarb, A.H. (2004). Anaerobic exercise and oxidative stress: A review. Canadian Journal of Applied Physiology, 29, 245-263.

Bloomer, R.J., Goldfarb, A.H., Wideman, L., Mckenzie, M.J., \& Consitt, L.A. (2005). Effects of acute aerobic and anaerobic exercise on blood markers of oxidative stress. Journal of Strength and Conditioning Research, 19, 276-285.

Clarkson, P.M., Kearns, A.K., Rouzier, P., Rubin, R., \& Thompson, P.D. (2006). Serum creatine kinase levels and renal function measures in exertional muscle damage. Medicine and Science in Sports and Exercise, 38, 623-627.

Cuevas, M.J., Almar, M., García-Glez, J.C., García-López, D., De Paz, J.A., Alvear-Ordenes, I., \& González-Gallego, J. (2005). Changes in oxidative stress markers and NF-kappa B activation induced by sprint exercise. Free Radical Research, 39, 431-439.

Demirba, R.,Yılmaz, R., Güzel, S., Çelik, H., Koçyigit, A., \& Özcan, E. (2006). Effects of treadmill exercise test on oxidative/antioxidative parameters and DNA damage. The Anatolian Journal of Cardiology, 6, 135-140.

Foley, J.M., Jayaraman, R.C., \& Prior, B.M. (1999). MRI measurements of muscle damage and adaptation after eccentric exercise. Journal of Applied Physiology, 87, 2311-2318.

Haff, G.G., Hobbs, R.T., Haff, E.E., Sands, W.A., Pierce, K.C., \& Stone, M.H. (2008). Cluster training: A novel method for introducing training program variation. Strength and Conditioning Journal, 30, 67-76.

Halliwell, B., \& Aruoma, O.I. (1991). DNA damage by oxygen-derived species. Its mechanism and measurement in mammalian system. FEBS Letter, 281, 9-19.

Hansen, K.T., Cronin, J.B., Pickering, S.L., \& Newton, M.J. (2011). Does cluster loading enhance lower body power development in preseason preparation of elite rugby players? Journal of Strength and Conditioning Research, $25,2118-2126$.

Houstis, N., Rosen, E.D., \& Lander, E.S. (2006). Reactive oxygen species have a causal role in multiple forms of insulin resistance. Nature, 440, 944-948.

Hulbert, A. (2005). On the importance of fatty acid composition of membranes for aging. Journal of Theoretical Biology, 234, 277-288.

Ji, L.L. (2000). Free radicals and antioxidants in exercise and sports. In W.E. Garrett \& D.T. Kirkendall (Eds.), Exercise and Sport Science. Philadelphia, PA: Lippincott Williams and Wilkins.

Kraemer, W.J., \& Fry, A.C. (1995). ACSM's guidelines for exercise testing and prescription (6 ${ }^{\text {th }}$ ed). Champaign, IL: Human Kinetics.

Kraemer, W.J., \& Ratamess, N.A. (2004). Fundamental of resistance training: Progression and exercise prescription. Medicine and Science in Sports and Exercise, 36, 674-688.

Lawton, T., Cronin, J., Drinkwater, E., Lindsell, R., \& Pyne, D. (2004). The effect of continuous repetition and intra-set rest training on bench press strength and power. Journal of Sports Medicine and Physical Fitness, 44, 361-367.

Lawton, T., Cronin, J., \& Lindsell, R. (2006). Effect of interrepetition rest intervals on weight training repetition power output. Journal of Strength and Conditioning Research, 20, 172-176.

MaClaren, D., \& Morton., J. (2012). Biochemistry for sport and exercise metabolism. Wiley-Blackwell. 
Margonis, K., Fatouros, I.G., Jamurtas, A.Z., Nikolaidis, M.G., Douroudos, I., \& Chatzinikolaou, A. (2007). Oxidative stress biomarkers responses to physical overtraining: Implications for diagnosis. Free Radical Biology and Medicine, 43, 901-910.

McAnulty, S.R., McAnulty, L.S., Nieman, D.C., Morrow, J.D., Utter, A.C., \& Dumke, C.L. (2005). Effect of resistance exercise and carbohydrate ingestion on oxidative stress. Free Radical Research, 39, 1219-1224.

Nikolaidis, M.G., \& Mougios, V. (2004). Effects of exercise on the fatty-acid composition of blood and tissue lipids. Sports Medicine, 34, 1051-1076.

Pillon, N.J., Croze, M.L., Vella, R.E., Soulère, L., Lagarde, M., \& Soulage, C.O. (2012). The lipidperoxidationbyproduct4-hydroxy-2-nonenal (4-HNE) induces insulin resistance in skeletal muscle through both carbonyl and oxidative stress. Endocrinology, 153, 2099-2111.

Pozzi, R., Rosa, J.C., Eguchi, R., Oller do Nascimento, C.M., Oyama, L.M., Aguiar, O. Jr., Chaves, M.D., \& Ribeiro, D.A. (2010). Genetic damage in multiple organs of acutely exercised rats. Cell Biochemistry and Function, 28, 632-636.

Quindry, J.C., Stone, W.L., King, J., \& Broeder, C.E. (2003). The effects of acute exercise on neutrophils and plasma oxidative stress. Medicine and Science in Sports and Exercise, 35, 1139-1145.

Radak, Z., \& Goto, S. (2000). Oxidative modification of proteins and DNA. In Z. Radak (Ed.), Free radicals in exercise and aging. Champaign, IL: Human Kinetics.

Radak, Z., Ogonovszky, H., Dubecz, J., Pavlik, G., Sasvari, M., Pucsok, J., Berkes, I., Csont, T., \& Ferdinandy, P. (2003). Super-marathon race increases serum and urinary nitrotyrosine and carbonyl levels. European Journal of Clinical Investigation, 33, 726-730.

Radak, Z., Pucsok, J., Meeseki, S., Csont, T., \& Ferdinandy, P. (1999). Muscle soreness-induced reduction in force generation is accompanied by increased nitric oxide content and DNA damage in human skeletal muscle. Free Radical Biology and Medicine, 26, 1059-1063.

Ramel, A., Wagner, K.H., \& Elmadfa, I. (2004). Plasma antioxidants and lipid oxidation after submaximal resistance exercise in men. European Journal of Nutrition, 43, 2-6.

Sakai, Y., Iwamura, Y., Hayashi, J., Yamamoto, N., Ohkoshi, N., \& Nagata, H. (1999). Acute exercise causes mitochondrial DNA deletion in rat skeletal muscle. Muscle Nerve, 22, 258-261.

Shephard, R.J., \& Shek, P.N. (1998). Association between physical activity and susceptibility to cancer: Possible mechanisms. Sports Medicine, 26, 293-315.

Uchida, K., \& Stadtman, E.R. (1992). Modification of histidine residues in proteins by reaction with 4-hydroxynonenal. PNAS, 89, 4544-4548.

Uchiyama, S., Tsukamoto, H., Yoshimura, S., \& Tamaki, T. (2006). Relationship between oxidative stress in muscle tissue and weight-lifting induced muscle damage. Pflugers Arch-European Journal of Physiology, 452, 109-116.

Weiss, L.W. (1991). The obtuse nature of muscular strength: The contribution of rest to its development and expression. Journal of Applied Sports Science and Research, 5, 219-227.

Whitehead, T.G., Thorpe, H.G., \& Maxwell, S. (1992). Enhanced chemiluminescent assay for antioxidant capacity in biological fluids. Analytica Chimica Acta, 226, 265-277.

Wierzba, T.H., Olek, R.A., Fedeli, D., \& Falcioni, G. (2006). Lymphocyte DNA damage in rats challenged with a single bout of strenuous exercise. Journal of Physiology and Pharmacology, 57(Suppl 10), 115-131.

Williams, S.L., Natalie, A.S., Louise, A.L., \& Jeff, S.C. (2006). Antioxidant requirements of endurance athletes: Implications for health. Nutrient Review, 64, 93-108.

Submitted: March 15, 2015

Accepted: July 21, 2015

Correspondence to:

Hamid Arazi, Ph.D.

Department of Exercise Physiology

Faculty of Sport Sciences, University of Guilan

P.O. Box: 41635-1438, Rasht, Iran

Tel: +98 911-1399207

Fax: +98 13-33690675

E-mail: hamidarazi@yahoo.com

\section{Acknowledgement}

The authors are grateful to the volleyball players and National Volleyball Federation for their contribution and cooperation. There is no financial support for this project. The results of this study do not constitute endorsement of any product by the authors or the National Strength and Conditioning Association. 